\title{
Spinal Gorham-Stout syndrome: radiological changes and spinal deformities
}

\author{
Chang-Zhi $\mathrm{Du}^{1}$, Song $\mathrm{Li}^{2}$, Liang Xu${ }^{2}$, Qing-Shuang Zhou ${ }^{1}$, Ze-Zhang Zhu ${ }^{2}$, Xu Sun ${ }^{1}$, Yong Qiu ${ }^{1}$ \\ ${ }^{1}$ Department of Spine Surgery, Nanjing Drum Tower Hospital, Clinical College of Nanjing Medical University, Nanjing 210008, China; \\ ${ }^{2}$ Department of Spine Surgery, Nanjing Drum Tower Hospital, Medical School of Nanjing University, Nanjing 210008, China
}

Correspondence to: Yong Qiu, MD; Xu Sun, MD. Department of Spine Surgery, Nanjing Drum Tower Hospital, Clinical College of Nanjing Medical University, Nanjing 210008, China. Email: scoliosis2002@sina.com; drsunxu@163.com.

\begin{abstract}
Background: Gorham-Stout syndrome (GSS) involving the spine is a rare clinical entity, and there is a paucity of comprehensive study on its radiological features. We aimed to present the radiological changes and spinal deformities in patients with spinal GSS.

Methods: From January 2005 to December 2017, 11 consecutive GSS patients with spinal deformity were identified. Their medical records and imaging features were retrospectively reviewed. Computed tomography (CT) and magnetic resonance imaging (MRI) were used for a precise evaluation of spinal involvement. Posteroanterior and lateral standing radiographs were used to evaluate the spinal deformity.

Results: CT showed multi-level generalized osteolytic lesions, with ill-defined fluid attenuation adjacent to the osseous changes. MRI demonstrated hyperintense signals on both T1- and T2-weighted images, while the unaffected segments showed normal signal intensity. Seven patients $(63.6 \%)$ had a dominant feature of kyphosis, and 4 (36.4\%) had scoliosis when spinal GSS was diagnosed. Kyphosis variably spanned from C7 to L1, averaged $94^{\circ}$ (range, $53^{\circ}$ to $158^{\circ}$ ), and was associated with sagittal imbalance in 4 cases. In our series, the apex of kyphosis and scoliosis coincided within the most seriously osteolytic segment. All patients received medication for GSS. Two adolescents taking Boston braces showed a relatively stable deformity. Four patients received long posterior spinal fusion, but two had fusion failure.

Conclusions: CT and MRI investigations are important in the initial diagnosis and continued management for spinal GSS. A typical spinal deformity secondary to GSS presents as kyphosis or kyphoscoliosis, which is usually highly variable but highly concordant with osteolysis in terms of span and apex.
\end{abstract}

Keywords: Gorham-Stout syndrome (GSS); radiological characteristic; spinal deformity; surgery

Submitted Dec 10, 2018. Accepted for publication Mar 22, 2019.

doi: 10.21037/qims.2019.03.17

View this article at: http://dx.doi.org/10.21037/qims.2019.03.17

\section{Introduction}

Gorham-Stout syndrome (GSS), characterized by spontaneous, aggressive and progressive osteolysis, is a rare condition of unknown cause (1-3). This disease occurs at any age, without a gender or race predilection $(1,4)$. GSS can affect any part of the skeleton, with the majority of bone lesions in maxillofacial areas, upper extremities (including the scapula), and the trunk (including the ribs, clavicles and pelvic girdles) (5). Radiologic investigations are important in the initial diagnosis, and the continued management and long-term follow-up of patients with GSS. The advent of computed tomography (CT) and magnetic resonance imaging (MRI) has led to a better understanding of this disease (6). In terms of treatment, it is mostly palliative, and always limited to symptom management. To date, there is still no known intervention which addresses GSS consistently and effectively, and all reported interventions are considered experimental treatments (7).

Due to the non-specificity of the early clinical 
manifestations of osteolysis, GSS patients with spinal involvement can continue daily activities until a pathological vertebral fracture or symptomatic spinal deformity (8). As osteolysis progresses, it may cause variable spinal deformities such as scoliosis, kyphosis, subluxation, or even dislocation of the spine (9). Sometimes, neurological deficits may occur due to the intra-spinal hematoma or severe vertebral dislocation secondary to spinal GSS $(10,11)$. If accompanied by the additional involvement of the thoracic cage, GSS patients with spinal deformity may experience chylothorax, restrictive ventilatory impairment, or lifethreatening complications (12-14). Therefore, it is crucial to offer early diagnosis and management for spinal GSS. Theoretically, for patients with a severe spinal deformity or neurological deficits, surgical strategies of lesion resection and bone reconstruction may lead to improved outcomes. However, spinal fusion in patients with GSS has been associated with a high incidence of graft resorption and fusion failure, requiring reoperation $(10,15)$. In general, a poor prognosis is usually associated with spinal GSS (1), and we should pay more attention to spinal deformity to prevent unfavourable adverse outcomes.

Approximately 50 cases of GSS with involvements of the spine have been reported in the literature $(9,16,17)$. However, the descriptions are mostly based on sporadic case reports. To the best of our knowledge, there have been no comprehensive studies of radiological changes and spinal deformity in patients with GSS. Moreover, the progression of spinal deformity in such patients has never been investigated in previous studies. Here, we present a consecutive series of 11 cases with GSS, who had the main complaint of progressive spinal deformity, with the aim of illustrating the radiologic characteristics and of exploring the progression and treatment of spinal deformity in patients with GSS.

\section{Methods}

\section{Patients}

After approval from the institutional review board of our hospital, we retrospectively reviewed the medical records of patients with spinal GSS who presented with a spinal deformity in our department from January 2005 to December 2017. Diagnosis of GSS was based on the positive histopathological examination after biopsy of the lesion and the radiologic evidence of progressive osteolysis after exclusion of infection, tumour, and metabolic or endocrine disorders $(18,19)$. Patients were excluded if they had incomplete data of medical records, laboratory tests, or radiological examinations. In total, 11 consecutive GSS patients with spinal deformity were enrolled in this study, including 6 females and 5 males. All patients were initially diagnosed with a spinal deformity by their orthopedists who referred them to our clinic for further assessment and treatment. Diagnosis of spinal GSS was not made until referral to our clinic.

\section{Radiologic characteristics}

All radiographic data were reviewed jointly by a senior orthopedic spine surgeon and a radiologist with experience in the diagnosis of musculoskeletal disease. The radiographic pattern of the spinal deformity was assessed on posteroanterior and lateral standing films. The type of scoliosis was defined according to the classification system proposed by the Scoliosis Research Society (20): cervicothoracic (apex at C7 or T1), thoracic (apex between T2 and T11), thoracolumbar (apex at T12 or L1), lumbar (apex between L2 and L4), and lumbosacral (apex at L5 or below). Patients were considered to have a C-shaped curve if they had a 1 major curve involving any region of the spine, an S-shaped curve if they had 2 separate curves, or a triple curve pattern if they had 3 separate curves. The span, apex, and convexity of these curves were also recorded. Coronal balance was measured as the deviation of the $\mathrm{C} 7$ plumb line from the center sacral vertical line, and was considered significant with a deviation of over $20 \mathrm{~mm}$. The parameters measured in the sagittal plane included segmental kyphosis and sagittal balance. Sagittal balance was determined according to the horizontal distance from the $\mathrm{C} 7$ plumb line to the posterior superior corner of S1, and imbalance was considered to be present if the distance surpassed $40 \mathrm{~mm}$ (21). CT scanning and three-dimensional reconstructions were used for precise evaluation of the range of osteolysis. MRI was used to define the extent of spinal canal encroachment, vascular formation, and soft tissue involvement (22).

\section{Statistical analysis}

Descriptive analysis was used for statistical analysis. All continuous data, if the sample was normally distributed, was expressed as the means \pm standard deviation; otherwise, the median and range were used. 


\section{Results}

In our series, there were 2 young children, 7 adolescents and 2 adults. Ten patients had an earlier average age at detection of spinal deformity than that at diagnosis of GSS $(13.9 \pm 13.5$ versus $16.5 \pm 13.1$ years $)$. The general data and clinical characteristics are shown in Table 1.

\section{Radiological changes}

Cortical loss and/or progressive osteolysis were identified in all patients. Spinal involvement was not clearly detected on plain radiographs in most cases. On CT scanning, spinal involvements were characterized by widely multilevel generalized osteolytic lesions, with ill-defined fluid attenuation adjacent to the osseous changes. MRI demonstrated hyperintense signals on both T1- and T2weighted images, while the unaffected segments showed normal signal intensity (Figure 1).

As shown in Table 2, the locations of the lesions were continuous and variable, being cervicothoracic in 2 patients, thoracic in 3 , thoracolumbar in 5 and thoracolumbosacral in 1 . Lesions were present in a varied span of vertebral levels from $\mathrm{C} 4$ to $\mathrm{S} 4$, and the median span was 10 segments (ranging from 6 to 16). The most commonly involved segments were the thoracic spine. Wedged vertebrae were detected in all patients, with a median segment of 3 (ranging from 2 to 7). The most seriously pathological fracture or highly wedged vertebra mainly occurred in the upper thoracic or thoracolumbar vertebrae (Figure 2).

Additionally, an infiltrative soft tissue abnormality adjacent to the area of osteolysis was identified in 7 patients (63.6\%), who presented with a hyperintense signal in T2weighted images and demonstrated an intense enhancement following administration of gadolinium contrast. Compression of the spinal cord or nerve root was detected in 6 patients $(54.5 \%)$. Syringomyelia was also identified in 1 patient (Patient 10).

\section{Characteristics of spinal deformities}

All patients were diagnosed with spinal GSS at their first visit to our clinic. There were 7 patients $(63.6 \%)$ with a main presentation of kyphosis and 4 (36.4\%) with scoliosis when GSS was diagnosed. The patterns of the spinal deformities were highly variable in terms of the scoliosis shape, curve location, and sagittal alignment. Table 3 shows the features of the spinal deformities in our cohort.
Among the 7 patients with mainly significant kyphosis (Patients 1, 2, 3, 5, 7, 9, and 10), 6 (85.7\%) had angular kyphosis and the remaining patient (Patient 7) had regular kyphosis. The span of the kyphosis varied from C7 to L1, with a median of 7 levels (ranging from 5 to 8 ). The thoracic spine was the most commonly involved segment, with the median apex at T7 (ranging from T3 to T11). The median angle of maximum kyphosis was $94^{\circ}$ (ranging from $53^{\circ}$ to $158^{\circ}$ ). Four patients (Patients $1,2,3$, and 10) showed severe sagittal imbalance, with a median distance (from the C7 plumb line to the posterior superior corner of S1) of $50.5 \mathrm{~mm}$ (ranging from 40 to $82 \mathrm{~mm}$ ). In the coronal plane, there were 6 cases of a C-shaped curve and only 1 of an S-shaped curve. The major Cobb angle of scoliosis was $34^{\circ}$ (ranging from $19^{\circ}$ to $72^{\circ}$ ); however, two patients were identified with coronal imbalance (Patients 3 and 9).

Four patients had a significant presentation of scoliosis (Patients 4, 6, 8, and 11). The curve localized to the thoracic/thoracolumbar in 1 case and the thoracolumbar/ lumbar in 2 of 3 cases with an S-shaped curve, while it was thoracolumbar in 1 case with a C-shaped curve. The median span of the curve was 6.5 levels (ranging from 6 to 11 levels), corresponding to a median apex at T12 (ranging from T5 to L3). The median Cobb angle of scoliosis was $40^{\circ}$ (ranging from $18^{\circ}$ to $61^{\circ}$ ), and all kyphoses were regular, with an angle of $34^{\circ}$ (ranging from $30^{\circ}$ to $41^{\circ}$ ). All 4 patients were well-balanced in the coronal and sagittal planes.

As shown in Figure 2, the span of spinal deformity showed a high concordance with the osteolysis lesions. The locations of the kyphoses were more consistent with the locations of lesions compared to those of scoliosis. Various spinal deformities were occasionally associated with pathological fractures or wedged vertebrae. The apex of kyphosis in 9 patients $(81.8 \%)$ was formed at the location where the most seriously pathological fracture or highly wedging vertebrae occurred, and such a phenomenon on scoliosis was only identified in 5 patients (45.5\%). Overall, kyphosis was more closely associated with widely multilevel osteolytic lesions.

\section{Management of GSS and spinal deformity}

As shown in Table 4, a variety of treatments was performed according to the patients' condition. Ten patients (except Patient 1) had an adequate radiographic follow-up after $3.9 \pm 1.5$ years (ranging from 2 to 7 years). Among these patients, medication, including bisphosphonates, and 


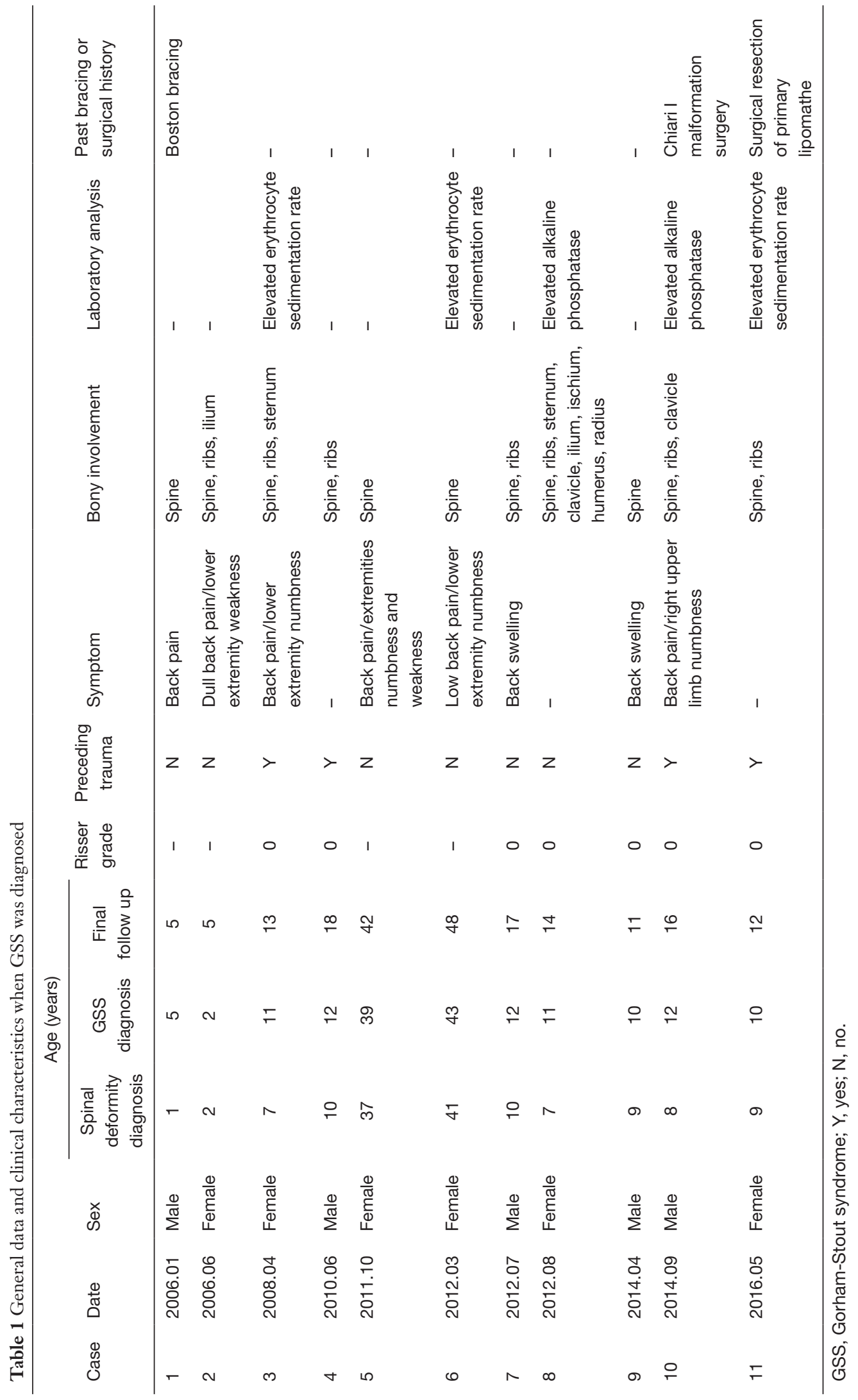



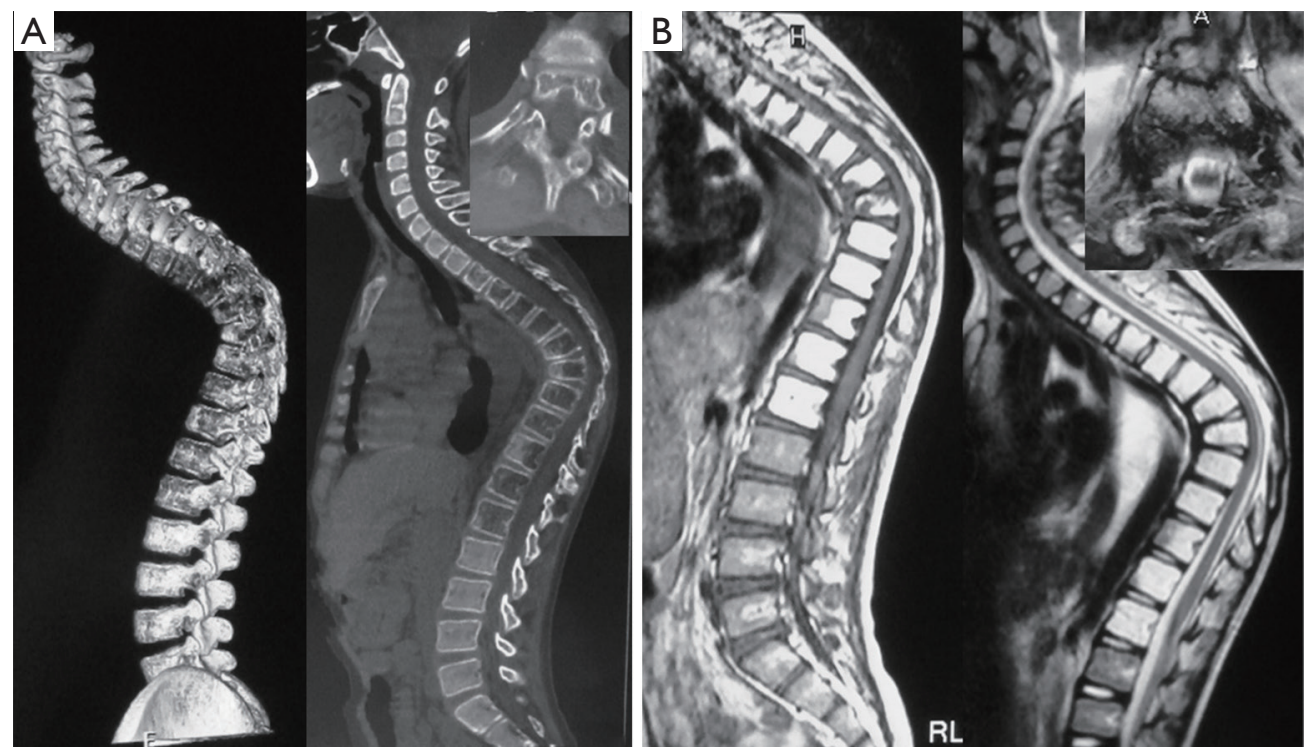

Figure 1 Patient 7, male, 12 years. (A) CT scan and three-dimensional reconstruction showing a lytic lesion in the multi-segment thoracic spine and destructive vertebral body lesions. (B) MRI showing hyperintense signal of the affected vertebrae on both T1- and T2-weighted sequences and severe narrowing of the canal at the apex of kyphosis. CT, computed tomography; MRI, magnetic resonance imaging.

Table 2 Imaging features of CT and MRI when GSS was diagnosed

\begin{tabular}{|c|c|c|c|c|c|c|c|}
\hline Case & $\begin{array}{c}\text { Span of } \\
\text { osteolysis [No.] }\end{array}$ & $\begin{array}{l}\text { Wedging of vertebral } \\
\text { body [No.] }\end{array}$ & $\begin{array}{l}\text { T1 weighted of } \\
\text { MRI }\end{array}$ & $\begin{array}{l}\text { T2 weighted } \\
\text { of } \mathrm{MRI}\end{array}$ & $\begin{array}{l}\text { Intraspinal } \\
\text { hematoma }\end{array}$ & $\begin{array}{l}\text { Spinal cord } \\
\text { or nerve } \\
\text { compression }\end{array}$ & $\begin{array}{c}\text { Paravertebral } \\
\text { muscle } \\
\text { involvement }\end{array}$ \\
\hline 2 & T6-L2 [9] & T9-T12 [4] & Hyperintense & Hyperintense & $\mathrm{N}$ & $\mathrm{Y}$ & $\mathrm{Y}$ \\
\hline 3 & C4-T7 [11] & T3-T5 [3] & Hyperintense & Hyperintense & $\mathrm{N}$ & $\mathrm{Y}$ & $\mathrm{N}$ \\
\hline 5 & T2-T7 [6] & T3-T5 [3] & Hyperintense & Hyperintense & $\mathrm{N}$ & $\mathrm{Y}$ & $\mathrm{Y}$ \\
\hline 6 & T8-L5 [10] & T11-T12, L3-L4 [4] & Hyperintense & Hyperintense & $\mathrm{Y}$ & $\mathrm{Y}$ & Y \\
\hline 7 & T3-L1 [11] & T7-T8 [2] & Hyperintense & Hyperintense & $\mathrm{N}$ & $\mathrm{N}$ & $\mathrm{Y}$ \\
\hline 8 & T10-L3 [6] & L1-L3 [3] & Hyperintense & Hyperintense & $\mathrm{N}$ & $\mathrm{N}$ & $\mathrm{N}$ \\
\hline 11 & T7-S4 [15] & T8-L2 [7] & Hyperintense & Hyperintense & $\mathrm{N}$ & $\mathrm{N}$ & $\mathrm{N}$ \\
\hline
\end{tabular}

CT, computed tomography; MRI, magnetic resonance imaging; GSS, Gorham-Stout syndrome; No., the number of involved vertebrae; $\mathrm{Y}$, yes; N, no.

calcium supplementation, was recommended for the treatment of spinal GSS; however, deterioration of osteolysis was confirmed in 2 patients (Patients 2 and 7). In terms of the spinal deformity, 2 adolescents (Patients 8 and 11) received regular Boston bracing, and the deformities stabilized in both. In addition, 4 patients [Patients 2, 4 (Figure 3), 5, and 9] received corrective surgery with a posterior instrumentation that skipped the involved 


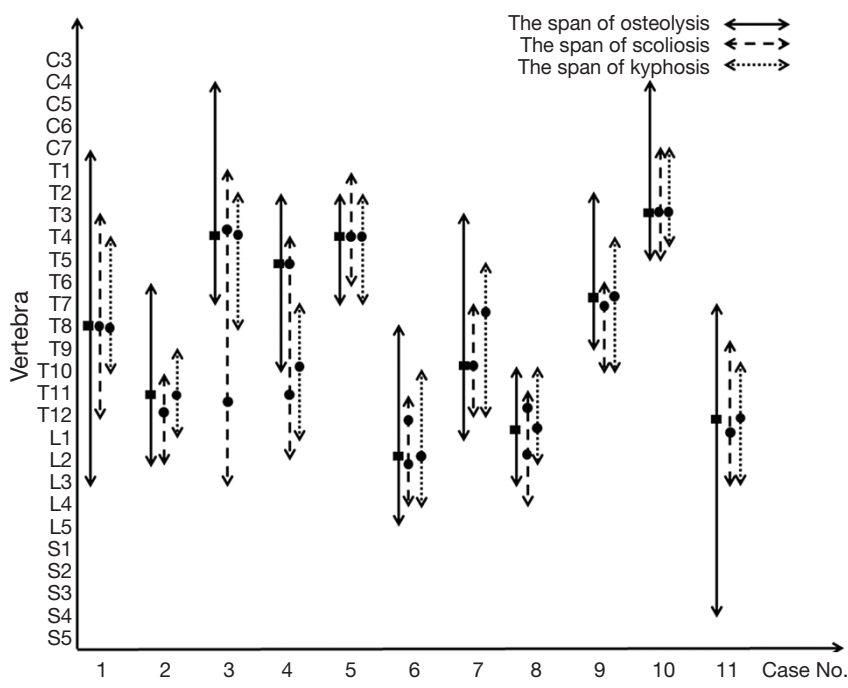

Figure 2 Span of osteolysis and spinal curves in each of the 11 patients when spinal Gorham disease was diagnosed in our clinic. The solid black rectangle indicates the most seriously pathological fractures or highly wedging vertebrae, and the solid black circle indicates the apical vertebra of scoliosis or kyphosis.

segments of GSS. However, Patient 2 developed kyphosis progression due to fusion failure and proximal junctional kyphosis but refused revision surgery, and Patient 5 resorted to revision surgery because of rod breakage (Figure 4). The remaining patients (Patients 3, 6, 7, and 10) refused the recommended surgery because of indeterminate prognosis or high surgical complications, and all of them significantly progressed in regards to their spinal deformity.

\section{Discussion}

The current study reported a rare condition of patients with spinal GSS. To our knowledge, this is the first comprehensive study of spinal GSS with the largest sample to date. In our study, osteolytic lesions were continuous but variable, with pathological fractures and wedged vertebrae. The patterns of the spinal deformity were highly variable in terms of the curve location, shape, apex, and sagittal alignment. Regardless, kyphosis or kyphoscoliosis presented as the typical deformity pattern in this disease, and the span and apex of kyphosis showed good concordance with that of progressive osteolysis. Our study also provided important data on treatment with medication, bracing, and surgical intervention, all of which tended to result in nearsatisfactory outcomes.

As reported both in our cases and in the literature
(9,16,23-29), and summarized in Table 5, spinal GSS can occur at any age and at any level from the cervical spine to the sacrum; however, the cervical and thoracic vertebrae are the most frequently involved regions. The early clinical manifestations of GSS are often subtle and nonspecific. Patients may present with pain and swelling following a trivial injury, or present with a long history of chronic dull pain $(8,9)$. The prognosis can be optimized if early diagnosis is confirmed during the course of the disease. As the etiology and precise pathogenesis are not well understood, diagnosis of GSS is challenging and often delayed. As in our series, all patients were misdiagnosed by their local orthopedists and then referred to our clinic generally until severe spinal deformity or neurological deficits had occurred (27).

Radiographic findings demonstrated partial or complete bony resorption involving one or more vertebrae. Subcortical osteolytic lesion was the early change, followed by progressive atrophy, fracture, and disappearance of a part of some vertebrae. CT scanning is useful in assessing the extent of bone destruction, but it cannot estimate the vascularity of the lesion. Moreover, 3D CT reconstructions can also be valuable for surgeons to analyze spinal morphology (30). The signal behavior varies on MRI because of the relative amount of vascular structures and fibrosis. Inflammation and increased capillary permeability can increase the signal intensity of MRI, and hypointense zones are due to fibrosis. Both in our and Dominguez et al.'s study (31), affected vertebrae showed hyperintensity on both T1- and T2-weighted images. Damron et al. (32) indicated that hyperintensity was closely associated with active Gorham-Stout disease. MRI is useful in differentiating between early, active, and later stages by demonstrating changes in signal intensity over time. Therefore, the role of MRI is not to provide a specific diagnosis but rather to demonstrate the progressive nature of this disorder.

However, no data are available on the risk factors for spinal deformity onset and progression. From the natural history provided by our cases, we believe that because of the subcortical osteolytic lesion in the asymmetrical vertebral areas, the original spinal abnormality, especially structural kyphosis, develops rapidly $(8,9)$. After revealing that the span and apex of the spinal deformity have a high concordance with vertebral osteolysis, we also believe that the most seriously pathological fracture or highly wedging vertebrae might be the initial onset of deformity. The affected paravertebral soft-tissue, particularly the paravertebral muscle, also has a substantial effect on the progression of spinal asymmetry deterioration (33). In 


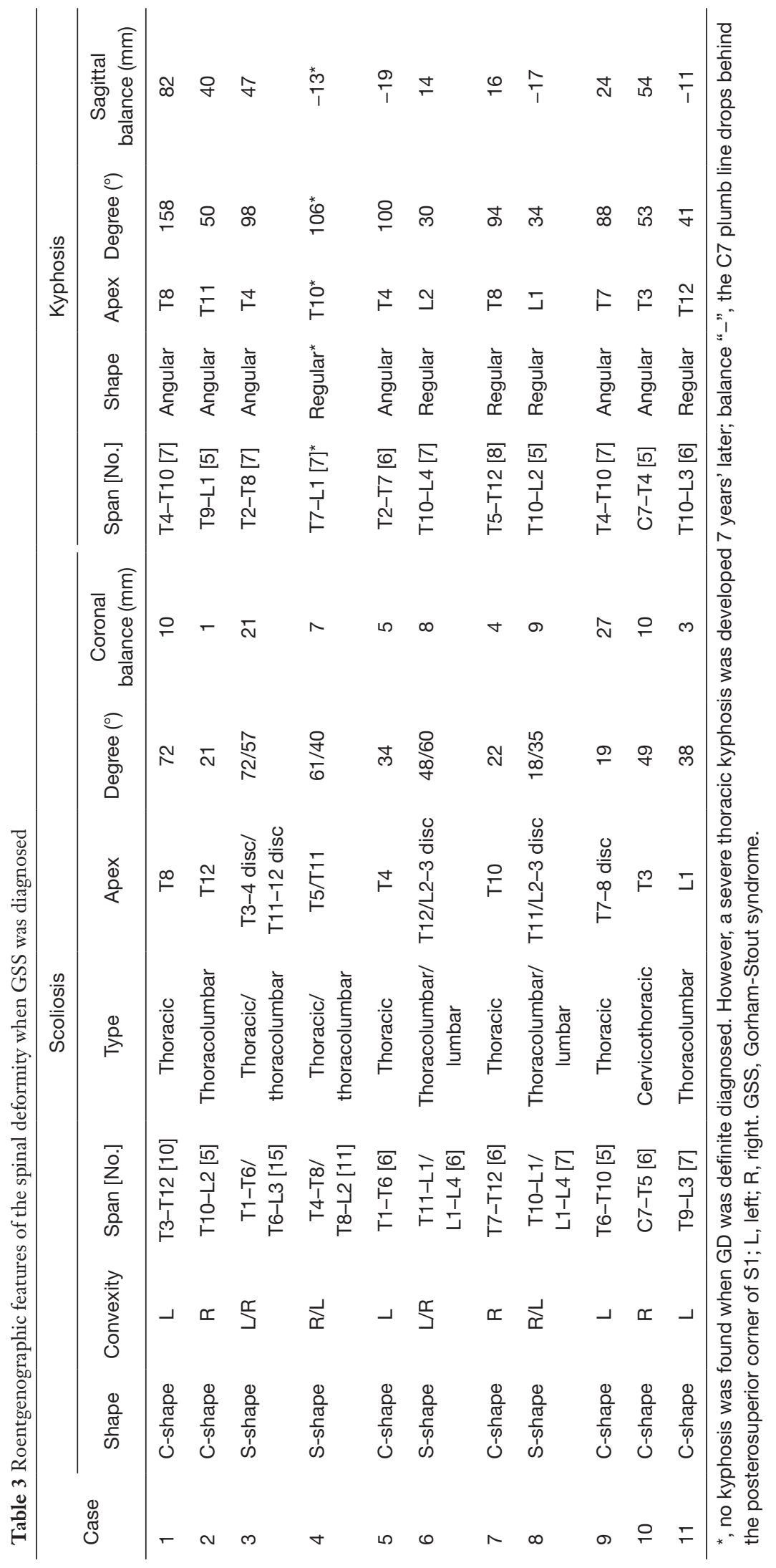




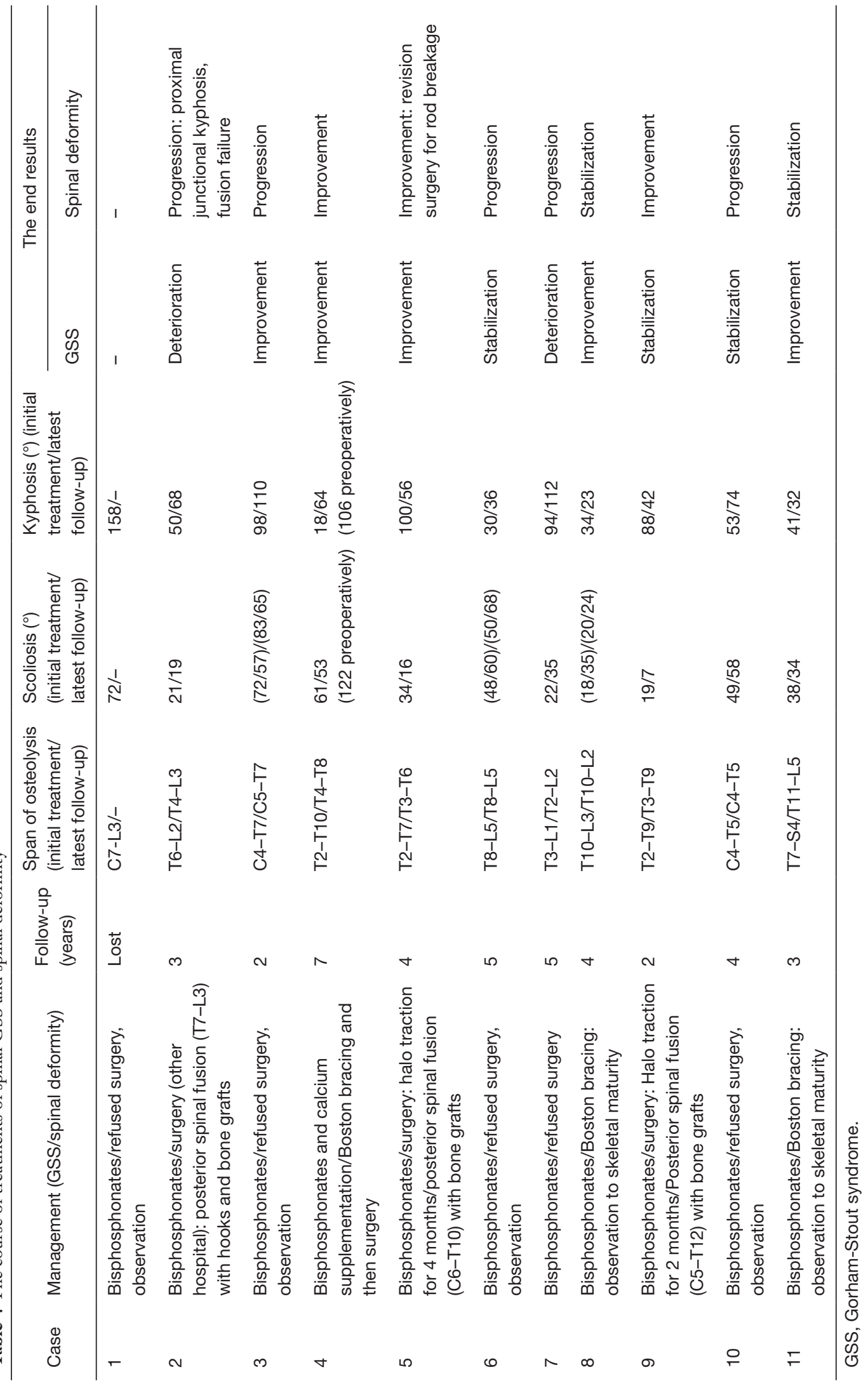



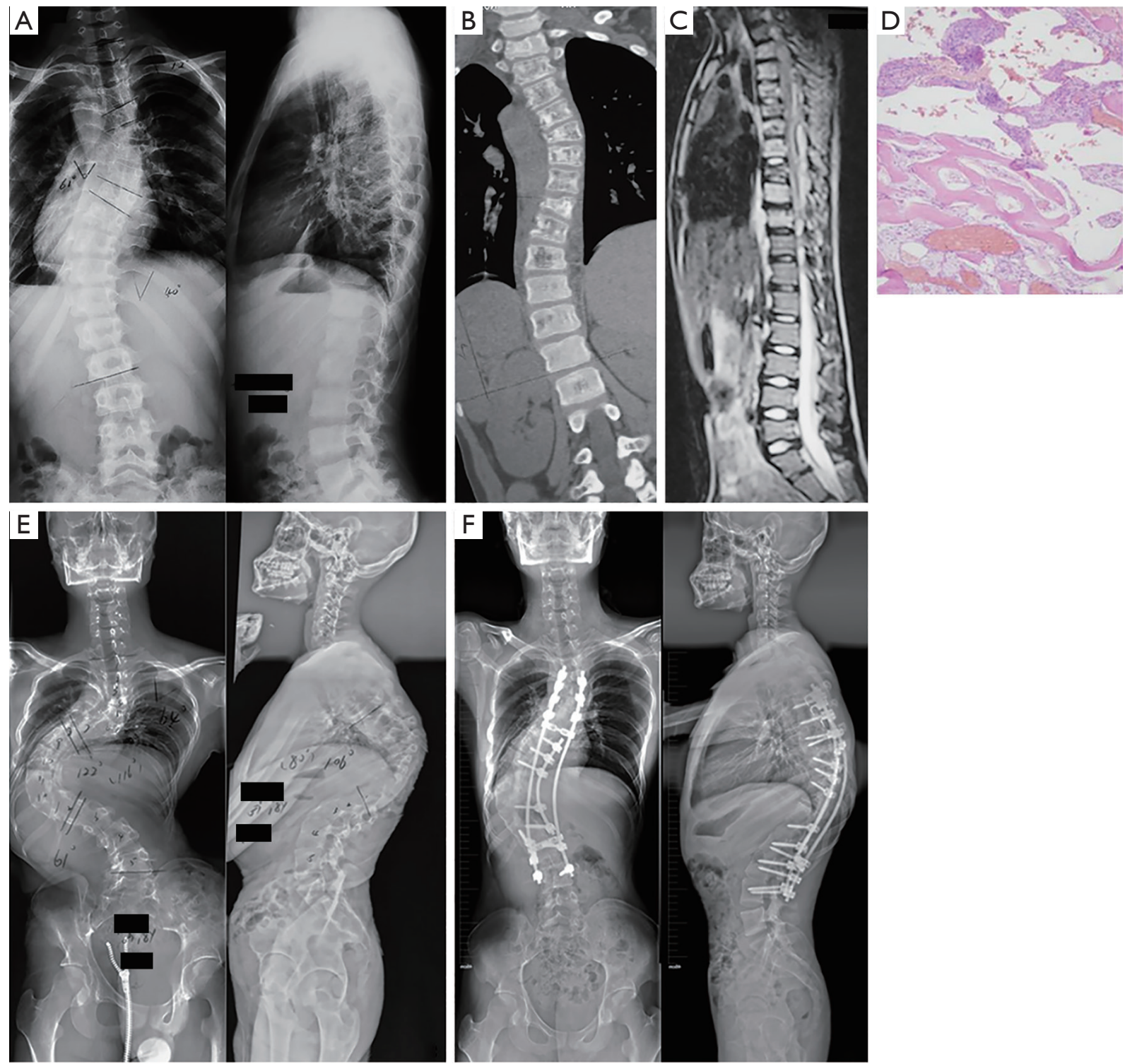

Figure 3 Patient 4, male, 12 years. (A) Initial X-rays showing C-shaped thoracolumbar scoliosis with a Cobb angle of 61. (B,C) CT scanning and MRI showing aggressive osteolysis and destructive lesions of the thoracic vertebra. (D) Hematoxylin and eosin staining showing thin-walled blood vessels with hemorrhage and scanty bone (magnification at 10x). (E) Corrective surgery was postponed because of the patient's extremely low bone mineral density, and was then replaced with Boston bracing. The compensatory thoracolumbar curve increased to $122^{\circ}$, with a severe thoracic kyphosis of $106^{\circ}$ and the apex at T10 7 years later. (F) Surgery with satellite rod and screws from T3 to L4 was performed after 2 months of Halo traction, and a significant improvement of the spinal deformity with rigid fusion was achieved. CT, computed tomography; MRI, magnetic resonance imaging.

addition, myelopathy and weakness of the paravertebral muscle can be caused by compression of the spinal cord and nerves associated with a serious pathological fracture and intra-spinal hematoma. For the above reasons, an affected patient may be predisposed to developing a typical deformity presenting with kyphosis or kyphoscoliosis. In some conditions, once the ribs and chest wall are involved, rapid and substantial curve progression can result in trunk shortening. Then, the unbalanced trunk can also trigger subluxation, dislocation or other extremely severe 

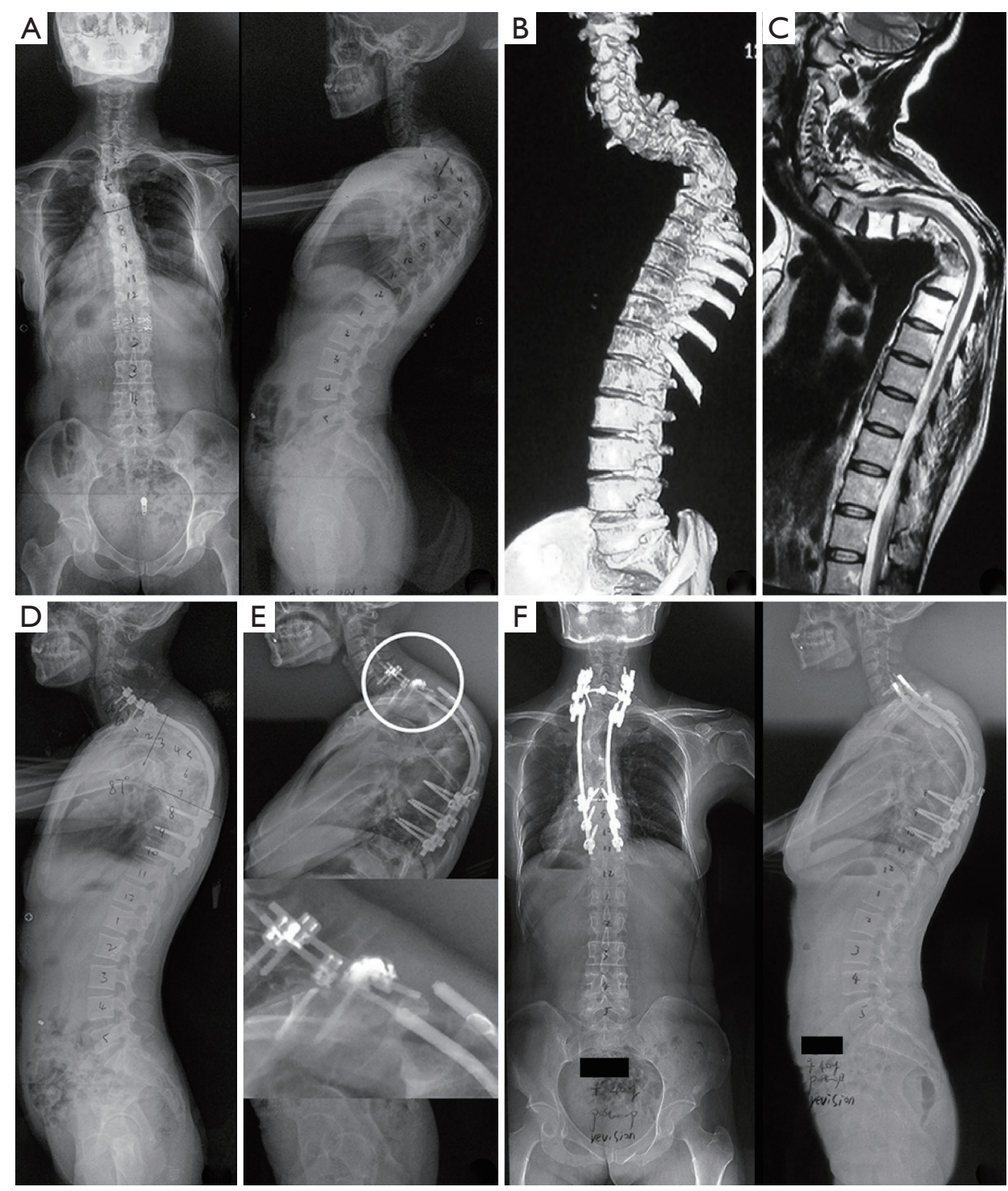

Figure 4 Patient 5, female, 39 years. (A) Preoperative X-rays showing cervicothoracic angular kyphosis with a curve of $100^{\circ}$. (B,C) CT and MRI demonstrating aggressive multiple lytic lesions on the thoracic vertebrae from T3 to T8. (D) Long posterior fixation was performed, with screws and rods from C6 to T10. (E) X-ray 14 months after fixation showing rod and screw breakage at the cervicothoracic junction. The white circle indicates the area of rod and screw breakage. $(\mathrm{F})$ The patient received a revision surgery, and no resorption of implanted bone or loss of correction was found during 3 years of follow-up. CT, computed tomography; MRI, magnetic resonance imaging.

deformities $(25,27)$. Consequently, asymmetrical osteolytic lesions with wedged vertebrae and affected paravertebral muscle contribute to the initial formation and deterioration of the spinal deformity.

Due to the lack of knowledge regarding this rare disease, spinal GSS is always misdiagnosed or missed when kyphosis or scoliosis is initially diagnosed. In our study, spinal deformity, especially thoracic kyphosis, rapidly deteriorated from the initial presentation. Most patients presented with primary significant kyphosis, and sagittal or coronal imbalance was also identified in such patients. Nevertheless, a relatively small Cobb angle and well-balanced trunk were both commonly observed in patients mainly presenting with scoliosis $(9,25,27)$. Similarly, most patients in previous sporadic case reports also showed remarkable thoracic kyphosis, with the apex on the most highly wedged 


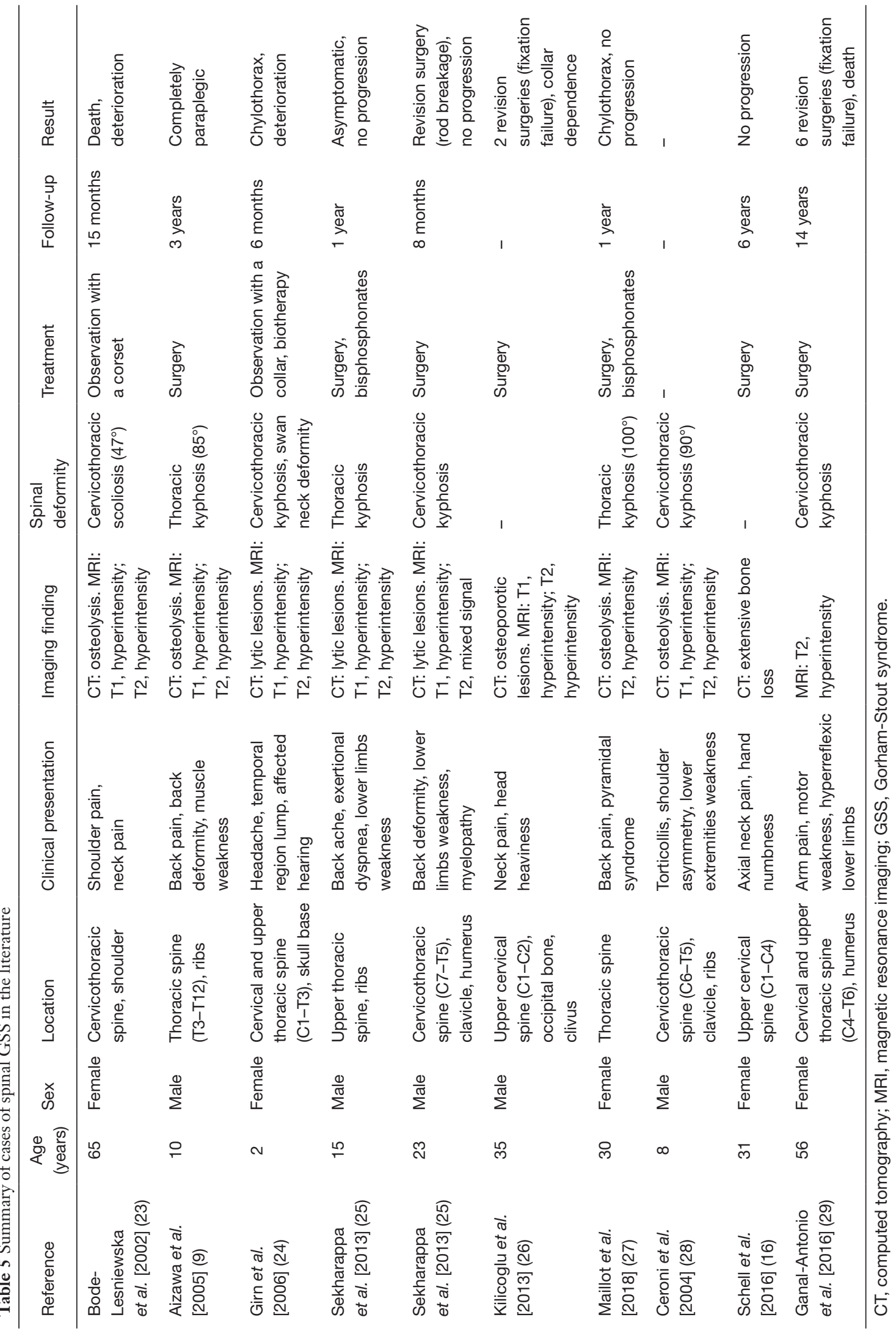


vertebrae $(27,28)$. Thus, kyphosis or kyphoscoliosis may be the typical spinal deformity secondary to spinal GSS. In addition, long C-curve type scoliosis was found early in this disease, and replaced with an S-shaped curve. Generally, a C-shaped curve is an initial injury, whereas an S-shaped curve may be an adaptive change in order to maintain trunk balance $(34,35)$. Overall, increased awareness of spinal GSS and earlier active management are of great importance for spinal deformity showing a progressive curve or an S-shaped curve tendency.

Thus far, there is no consensus regarding spinal GSS treatment with medication, radiotherapy, and surgery $(8,36-38)$. Since it is self-limiting, waiting to observe spontaneous arrest is also an appropriate option (38). In terms of treatment for an associated spinal deformity, we believe that a mild asymptomatic deformity can be stabilized by bracing. In a previous study (39), cervicothoracic kyphosis was also controlled with a brace. For a severe unstable deformity, surgical treatment may provide the best chance when possible. At this stage, brace treatment is usually useless and corrective surgery may be the only way to maintain the stability. Therefore, even though spinal GSS can be treated effectively, spinal deformity will continue to progress over time once severe imbalanced kyphosis or scoliosis develops. In addition, surgical interventions are frequently interrupted due to grievous multi-level osteolysis and missing bone substance for internal fixation. To avoid fixation pullout, pedicle screw fixation bridging the diseased vertebra with a correction rate of $50 \%$ to $60 \%$ is recommended. Unfortunately, as occurred in Sekharappa et al. (25), rod breakage at the cervicothoracic junction occurred in our patient. Ganal-Antonio et al. (29) reported a middle-aged female patient who underwent 6 revision surgeries for proximal junction kyphosis, broken nail, decoupling, distal junction kyphosis, screw extraction, and other reasons during the 14 years of follow-up. The fusion segment also gradually extended from the initial C5-T3 to the occipital bone-L3, and the patient eventually died of sepsis after limb paralysis and dependence on ventilator. Moreover, fusion failure and graft resorption were also identified in our study and a previous study (9). Therefore, the treatment of this rare condition is still full of challenge and unknowns.

\section{Limitations}

This study still has several limitations. First, there is still no consistently effective treatment of spinal GSS due to its unknown mechanism. Second, since most patients refused surgery, the current data might not be sufficient to judge the efficacy of surgical intervention, and the risks of severe complications associated with correction surgery remain undefined.

\section{Conclusions}

CT and MRI investigations play an important role in the initial diagnosis and continued management of spinal GSS. Kyphosis or kyphoscoliosis may be a typical spinal deformity. The spinal deformities deteriorate rapidly, and asymmetrical osteolytic lesions, highly wedged vertebrae, and affected paravertebral muscles might be the cause of progressive spinal deformity.

\section{Acknowledgements}

Funding: This work was supported by the National Natural Science Foundation of China (grant No. 81772422), the Postgraduate Research \& Practice Innovation Program of Jiangsu Province (grant No. KYCX18_1502), and Jiangsu Provincial Key Medical Center.

\section{Footnote}

Conflicts of Interest: The authors have no conflicts of interest to declare.

Ethical Statement: The approval for this study was obtained from the institutional review board of our hospital. Informed consent was waived due to the retrospective nature of this study.

\section{References}

1. Patel DV. Gorham's disease or massive osteolysis. Clin Med Res 2005;3:65-74.

2. Gorham LW, Stout AP. Massive osteolysis (acute spontaneous absorption of bone, phantom bone, disappearing bone); its relation to hemangiomatosis. J Bone Joint Surg Am 1955;37-A:985-1004.

3. Gowin W, Rahmanzadeh R. Radiologic diagnosis of massive idiopathic osteolysis (Gorham-Stout syndrome). Rontgenpraxis 1985;38:128-34.

4. Avelar RL, Martins VB, Antunes AA, de Oliveira Neto PJ, Andrade ES. Use of zoledronic acid in the treatment of Gorham's disease. Int J Pediatr Otorhinolaryngol 
2010;74:319-22.

5. Morimoto N, Ogiwara H, Miyazaki O, Kitamuara M, Nishina S, Nakazawa A, Maekawa T, Morota N. GorhamStout syndrome affecting the temporal bone with cerebrospinal fluid leakage. Int J Pediatr Otorhinolaryngol 2013;77:1596-600.

6. Manisali M, Ozaksoy D. Gorham disease: correlation of MR findings with histopathologic changes. Eur Radiol 1998;8:1647-50.

7. Heyd R, Rabeneck D, Dörnenburg O, Tselis N, Zamboglou N. Gorham-Stout syndrome of the pelvic girdle treated by radiation therapy: a case report. Strahlenther Onkol 2011;187:140-3.

8. Livesley PJ, Saifuddin A, Webb PJ, Mitchell N, Ramani P. Gorham's disease of the spine. Skeletal Radiol 1996;25:403-5.

9. Aizawa T, Sato T, Kokubun S. Gorham disease of the spine: a case report and treatment strategies for this enigmatic bone disease. Tohoku J Exp Med 2005;205:187-96.

10. Chong Ng L, Sell P. Gorham disease of the cervical spine-a case report and review of the literature. Spine 2003;28:E355-8.

11. Drewry GR, Sutterlin CE 3rd, Martinez CR, Brantley SG. Gorham disease of the spine. Spine 1994;19:2213-22.

12. Huang SY, Lee YM, Tzeng ST, Su CP, Huang SF, Wu YK, Lan CC. Gorham syndrome with postoperative respiratory failure and requiring prolonged mechanical ventilation. Respir Care 2013;58:e144-8.

13. Flörchinger A, Böttger E, Claass-Böttger F, Georgi M, Harms J. Gorham-Stout syndrome of the spine. Case report and review of the literature. Rofo 1998;168:68-76.

14. Kose M, Pekcan S, Dogru D, Akyuz C, Ozcelik U, Ozsurekci Y, Gulhan B, Demircin M, Kiper N. GorhamStout Syndrome with chylothorax: successful remission by interferon alpha-2b. Pediatr Pulmonol 2009;44:613-5.

15. Woodward HR, Chan DP, Lee J. Massive osteolysis of the cervical spine. A case report of bone graft failure. Spine 1981;6:545-9.

16. Schell A, Rhee JM, Allen A, Andras L, Zhou F. Surgical management of Gorham disease involving the upper cervical spine with occipito-cervical-thoracic fusion. a case report. Spine J 2016;16:e467-72.

17. Lala S, Mulliken JB, Alomari AI, Fishman SJ, Kozakewich HP, Chaudry G. Gorham-Stout disease and generalized lymphatic anomaly — clinical, radiologic, and histologic differentiation. Skeletal Radiol 2013;42:917-24.

18. Heffez L, Doku HC, Carter BL, Feeney JE. Perspectives on massive osteolysis. Report of a case and review of the literature. Oral Surg Oral Med Oral Pathol 1983;55:331-43.

19. Wáng YXJ, Santiago FR, Deng M, Nogueira-Barbosa MH. Identifying osteoporotic vertebral endplate and cortex fractures. Quant Imaging Med Surg 2017;7:555-91.

20. The Terminology Committee of the Scoliosis Research Society. A glossary of scoliosis terms. Spine 1976;1:57-8.

21. Yang X, Song Y, Liu L, Zhou C, Zhou Z, Wang L, Wang L. Emerging S-shaped curves in congenital scoliosis after hemivertebra resection and short segmental fusion. Spine J 2016;16:1214-20.

22. Martín Noguerol T, Barousse R, Socolovsky M, Luna A. Quantitative magnetic resonance (MR) neurography for evaluation of peripheral nerves and plexus injuries. Quant Imaging Med Surg 2017;7:398-421.

23. Bode-Lesniewska B, von Hochstetter A, Exner GU, Hodler J. Gorham-Stout disease of the shoulder girdle and cervico-thoracic spine: fatal course in a 65 -year-old woman. Skeletal Radiology 2002;31:724-9.

24. Girn HR, Towns G, Chumas P, Holland P, Chakrabarty A. Gorham's disease of skull base and cervical spine confusing picture in a two year old. Acta Neurochirurgica 2006;148:909-13.

25. Sekharappa V, Arockiaraj J, Amritanand R, Krishnan V, David KS, David SG. Gorham's Disease of Spine. Asian Spine J 2013;7:242-7.

26. Kilicoglu ZG, Kizildemir Kis N, Vardar Aker F, Berkman MZ, Simsek MM. Gorham disease of the craniocervical junction: X-ray, computed tomography, and magnetic resonance imaging findings. Spine J 2013;13:e11-4.

27. Maillot C, Cloche T, Le Huec JC. Thoracic osteotomy for Gorham-Stout disease of the spine: a case report and literature review. Eur Spine J 2018;27:2285-90.

28. Ceroni D, De Coulon G, Regusci M, Kaelin A. GorhamStout disease of costo-vertebral localization: radiographic, scintigraphic, computed tomography, and magnetic resonance imaging findings. Acta Radiol 2004;45:464-8.

29. Ganal-Antonio AK, Samartzis D, Bow C, Cheung KM, Luk KD, Wong YW. Disappearing bone disease of the humerus and the cervico-thoracic spine: a case report with 42-year follow-up. Spine J 2016;16:e67-75.

30. Spieth ME, Greenspan A, Forrester DM, Ansari AN, Kimura RL, Gleason-Jordan I. Gorham's disease of the radius: radiographic, scintigraphic, and MRI findings with pathologic correlation: A case report and review of the literature. Skeletal Radiol 1997;26:659-63.

31. Dominguez R, Washowich TL. Gorham's disease or vanishing bone disease: plain film, CT and MRI findings in two cases. Pediatr Radiol 1994;24:316-8. 
32. Damron TA, Brodke DS, Heiner JP, Swan JS, DeSouky S. Case report 803: Gorham's disease (Gorham-Stout syndrome) of scapula. Skeletal Radiol 1993;22:464-7.

33. Shah PB, Zasloff MA, Drummond D, Kaplan FS. Spinal deformity in patients who have fibrodysplasia ossificans progressiva. J Bone Joint Surg Am 1994;76:1442-50.

34. Nakamura Y, Nagai T, Iida T, Ozeki S, Nohara Y. Epidemiological aspects of scoliosis in a cohort of Japanese patients with Prader-Willi syndrome. Spine J 2009;9:809-16.

35. Nakamura Y, Murakami N, Iida T, Ozeki S, Asano S, Nohara Y, Nagai T. The characteristics of scoliosis in Prader-Willi syndrome (PWS): analysis of 58 scoliosis patients with PWS. J Orthop Sci 2015;20:17-22.

36. Lehmann G, Pfeil A, Böttcher J, Kaiser WA, Füller J, Hein G, Wolf G. Benefit of a 17-year long-term bisphosphonate therapy in a patient with Gorham-Stout syndrome. Arch Orthop Trauma Surg 2009;129:967-72.

37. Heyd R, Micke O, Surholt C, Berger B, Martini C, Füller J, Schimpke T, Seegenschmiedt MH, German Cooperative Group on Radiotherapy for Benign Diseases (GCG-BD). Radiation therapy for Gorham-Stout syndrome: results of a national patterns-of-care study and literature review. Int J Radiat Oncol Biol Phys 2011;81:e179-85.

38. Turra S, Gigante C, Scapinelli R. A 20-year follow-up study of a case of surgically treated massive osteolysis. Clin Orthop Relat Res 1990;250:297-302.

39. Heyden G, Kindblom LG, Nielsen JM. Disappearing bone disease: A clinical and histological study. J Bone Joint Surg Am 1977;59:57-61.
Cite this article as: $\mathrm{Du} \mathrm{CZ}, \mathrm{Li} \mathrm{S}, \mathrm{Xu} \mathrm{L}$, Zhou QS, Zhu ZZ, Sun X, Qiu Y. Spinal Gorham-Stout syndrome: radiological changes and spinal deformities. Quant Imaging Med Surg 2019;9(4):565-578. doi: 10.21037/qims.2019.03.17 